\title{
Geometry of Multi-layer Freeform Structures for Architecture
}

\author{
Helmut Pottmann* \\ TU Wien
}

\author{
Yang Liu ${ }^{\dagger}$ \\ TU Wien / Univ. of Hong Kong
}

\author{
Johannes Wallner \\ TU Graz
}

\author{
Alexander Bobenko ${ }^{\S}$ \\ TU Berlin
}

\author{
Wenping Wang ${ }^{\dagger}$ \\ Univ. of Hong Kong
}

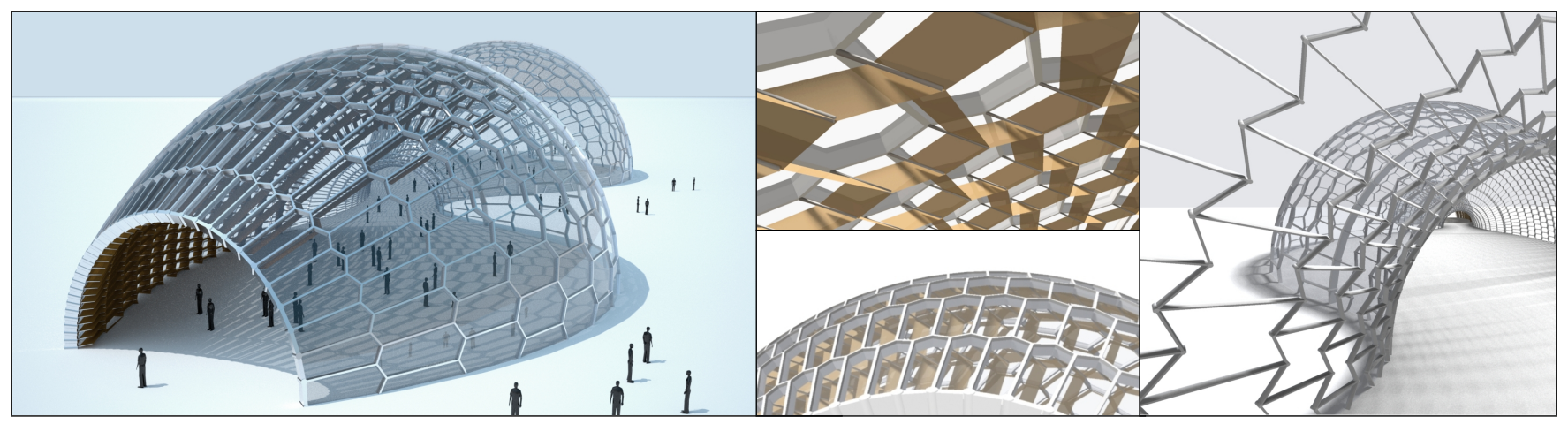

Figure 1: This architectural free form structure - built of beams of constant height meeting in optimized nodes and covered with planar glass facets - was designed using the theory and algorithms presented in this paper. Our method also allows for the construction of secondary parallel offsets at a variable distance, here physically realized as a structure designed to cast shadows which is optimized to reduce heat load for particular sun positions.

\section{Abstract}

The geometric challenges in the architectural design of freeform shapes come mainly from the physical realization of beams and nodes. We approach them via the concept of parallel meshes, and present methods of computation and optimization. We discuss planar faces, beams of controlled height, node geometry, and multilayer constructions. Beams of constant height are achieved with the new type of edge offset meshes. Mesh parallelism is also the main ingredient in a novel discrete theory of curvatures. These methods are applied to the construction of quadrilateral, pentagonal and hexagonal meshes, discrete minimal surfaces, discrete constant mean curvature surfaces, and their geometric transforms. We show how to design geometrically optimal shapes, and how to find a meaningful meshing and beam layout for existing shapes.

CR Categories: I.3.5 [Computer Graphics]: Computational Geometry and Object Modeling-Geometric algorithms, languages, and systems; I.3.5 [Computer Graphics]: Computational Geometry and Object Modeling-Curve, surface, solid, and object representations

Keywords: discrete differential geometry, surfaces in architecture, offset mesh, support structure, multi-layer construction, parallel mesh, curvatures, edge offset, hexagonal mesh, Koebe polyhedron.

\footnotetext{
*email: pottmann@geometrie.tuwien.ac.at

†email: \{yliu,wenping\}@cs.hku.hk

¥ email: j.wallner@tugraz.at

§email: bobenko@math.tu-berlin.de
}

\section{ACM Reference Format}

Pottmann, H., Liu, Y., Wallner, J., Bobenko, A., Wang, W. 2007. Geometry of Multi-layer Freeform Structures for Architecture. ACM Trans. Graph. 26, 3, Article 65 (July 2007), 11 pages. DOI = 10.1145/1239451.1239516 http://doi.acm.org/10.1145/1239451.1239516.

\section{Copyright Notice}

Permission to make digital or hard copies of part or all of this work for personal or classroom use is granted without fee provided that copies are not made or distributed for profit or direct commercial advantage and that copies show this notice on the first page or initial screen of a display along with the full citation. Copyrights for components of this work owned by others than ACM must be honored. Abstracting with credit is permitted. To copy otherwise, to republish, to post on servers, to redistribute to lists, or to use any component of this work in other works requires prior specific permission and/or a fee. Permissions may be requested from Publications Dept., ACM, Inc., 2 Penn Plaza, Suite 701, New York, NY 10121-0701, fax + (212) 869-0481, or permissions @ acm.org.

$10.1145 / 1239451.1239516$

http://doi.acm.org/10.1145/1239451.1239516

\section{Introduction}

Freeform shapes in architecture is an area of great engineering challenges and novel design ideas. Obviously the design process, which involves shape, feasible segmentation into discrete parts, functionality, materials, statics, and cost, at every stage benefits from a complete knowledge of the complex interrelations between geometry requirements and available degrees of freedom. Triangle meshes - the most basic, convenient, and structurally stable way of representing a smooth shape in a discrete way - do not support desirable properties of meshes relevant to building construction (most importantly, "torsion-free" nodes). Alternatives, namely quad-dominant and hexagonal meshes tend to have less weight, and can be constructed with geometrically optimized nodes and beams. However, the geometry of such meshes is more difficult. Especially challenging are aesthetic layout of edges and the geometric constraints of planar faces and optimized nodes.

Only recently, researchers have become interested in the geometric basics of single- and multilayer freeform structures which are not based on triangle meshes. Existing literature has been motivated by problems in the fabrication of steel/glass and other structures and mostly aims at the realizations of freeform shapes by meshes with planar faces [Glymph et al. 2002; Schober 2003; Cutler and Whiting 2007; Liu et al. 2006]. The latter paper introduced conical meshes which have planar faces and possess offset meshes at constant face-face distance from the base mesh. They can serve as the basis of multi-layer constructions, and so for the first time the problem of multilayered realization of a freeform surface by means of planar parts was solved in principle.

Until now the wealth of interesting geometry relevant to the construction of freeform structures in architecture has been explored only to a small extent. It is the aim of the present paper to show how the local structure of single- and multi-layer constructions can be analyzed with mesh parallelism as the main tool. This concept allows us to encode the existence of node axes and offsets in a discrete Gauss image, and to define discrete curvatures in a natural way. Optimization in the linear space of meshes parallel to a given mesh yields a modeling tool. A particularly important and interesting type of meshes are those possessing edge offsets. 

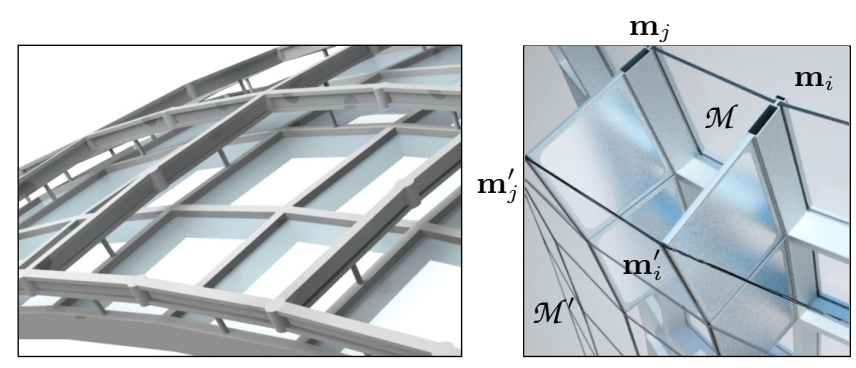

Figure 2: Multi-layer constructions based on the geometric support structure defined by two parallel meshes $\mathcal{M}, \mathcal{M}^{\prime}$ at approximately constant distance. On the left, the lower layer of the glass roof is suspended from the upper layer which has a structural function. The right hand image shows a rudimentary construction of a glass facade where the closed space between layers has an insulating function.

We show how mesh parallelism establishes a connection between meshes with edge offsets and Koebe polyhedra. Thus the research presented here is situated at the meeting point of discrete differential geometry, modeling, geometry processing, and architectural design.

Previous work in discrete differential geometry. Most of the work relevant to the present study concerns quadrilateral meshes with planar faces, which discretize so-called conjugate curve networks on surfaces [Sauer 1970]. They are a basic concept in the integrable system viewpoint of discrete differential geometry [Bobenko and Suris 2005]. Both the circular meshes and the conical meshes of Liu et al. [2006] are special cases which possess particularly nice geometric properties, and which correspond to those conjugate curve networks which are also orthogonal, i.e., the principal curvature lines. For convergence of such meshes to the network of principal curvature lines, see [Bobenko and Suris 2005]. The fact that principal curvature lines are a concept of Lie sphere geometry has a discrete manifestation in the unified treatment of circular and conical meshes as principal meshes of Lie sphere geometry [Bobenko and Suris 2007]. Elementary relations between circular and conical meshes, and meshes which enjoy both properties are discussed by Pottmann and Wallner [2007]. Special cases of the parallel meshes which are the topic of the present paper have been considered by [Sauer 1970]. Our work on curvature in the present paper extends results of Schief [2006], who defined a mean and Gaussian curvature for circular meshes via surface areas of discrete offset surfaces. That method apparently has first been applied to simplicial surfaces by Nishikawa et al. [1998] in a different context. Another classical definition of the mean curvature through variation of surface area of simplicial surfaces has been investigated by Polthier [2002a]. Discrete minimal surfaces realized as circular and conical meshes are the topic of several contributions (e.g. [Bobenko and Pinkall 1996; Bobenko et al. 2006]). Attempts to construct discrete minimal surfaces with planar quad meshes have been made by [Polthier 2002b]. The discrete minimal surfaces of [Bobenko et al. 2006] are particularly interesting because they provide a class of polyhedral surfaces with the edge offset property introduced in the present paper. It turns out in Section 3.2 that the edge offset property is closely related to results on orthogonal circle patterns [Schramm 1997; Bobenko and Springborn 2004].

Previous work in geometry processing. Only some of the papers mentioned above address the computation of the meshes they deal with [Bobenko and Springborn 2004; Bobenko et al. 2006; Liu et al. 2006]. The latter paper demonstrates how to design meshes with planar faces, circular meshes, and conical meshes by subdivision and optimization, and also how to approximate a given shape by a circular or conical mesh. As input for such mesh optimization algorithms any mesh aligned along a network of principal curvature lines may be used (see e.g. [Alliez et al. 2003; Tong et al. 2006]). Approximation of smooth surfaces by meshes with planar faces, without a focus on support structures and multilayer constructions can be achieved by variational shape approximation [Cohen-Steiner et al. 2004]. Cutler and Whiting [2007] modified this method with regard to aesthetics and architectural design. More generally, various research projects on geometry for architecture in general are promoted by the Smart Geometry group [SG].

Meshes whose faces are mostly planar 5-gons or 6-gons have certain desirable properties, but such meshes have received considerably less attention in the graphics community than triangle meshes and quad meshes. Some notable exceptions are papers on refinement processes by Akleman et al. [2005], and on combined primal/ dual subdivision [Oswald and Schröder 2003]. However, they do not consider planarity of faces or other aspects relevant to building construction.

\section{Mesh parallelism for architecture}

\subsection{Motivation and introduction}

Glass panels and multilayer metal sheets for roofing structures are planar as a rule, with only a few exceptions. The reason for this is mostly the prohibitive cost of manufacturing them otherwise. Obviously, every implementation of a freeform shape in terms of flat primitives faces the problem of approximating the given surface by a mesh with planar faces. If triangle meshes are employed, the geometry part of the solution of this problem consists of choosing the vertices and deciding which vertices to connect by edges. If faces can have more than three vertices, this approximation task is not so simple, because the condition of planar faces is no longer fulfilled automatically. It should be mentioned that also statics is simpler if we stay with triangle meshes. There are, however, the following issues which make other solutions attractive:

- In a steel/glass or other construction based on a triangle mesh, typically six beams meet in a node. This means a significantly higher node complexity compared to other types of meshes (see Fig. 4, right).

- Experience shows that the per area cost of triangular glass panels is higher than that of quadrilateral panels. This is mainly due to the fact that quadrilaterals fill their smallest rectangular bounding boxes better than triangles do.

- Generally one aims at less steel, more glass, and less weight, which also suggests the use of non-triangular faces.

- For the actual construction, torsion-free nodes are preferred. For this concept, see Figure 4 and the text below. The geometric theory however tells us that for triangle meshes in general torsionfree nodes do not exist.

- Apart from trivial cases, triangle meshes do not possess offsets at constant face-face or edge-edge distance; neither is it possible to use triangle meshes as basis of a multilayer construction where only the basic requirement of parallelity of layers is imposed.

This section shows how the concept of parallelism, which applies to meshes with planar faces, can be used to gain a unified view of these issues, especially geometrically optimal nodes and offset properties [Brell-Cokcan and Pottmann 2006]. Consider two meshes $\mathcal{M}$ and $\mathcal{M}^{\prime}$ which are combinatorially equivalent, i.e., there is a 1-1 correspondence between vertices and edges. We call the meshes $\mathcal{M}, \mathcal{M}^{\prime}$ parallel, if corresponding edges are parallel (see Figure 3). Before we consider parallel meshes from the mathematical viewpoint, we first describe some geometric problems connected with discrete surfaces in architecture where this concept occurs naturally. 


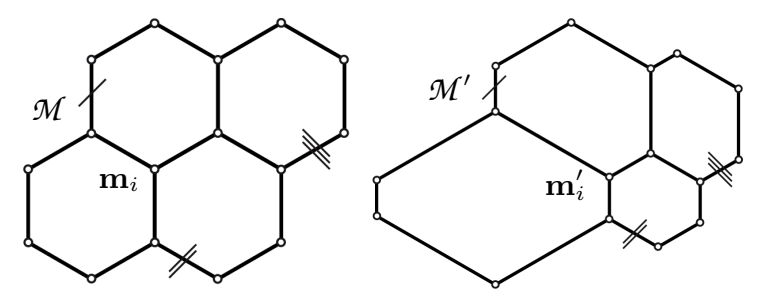

Figure 3: Meshes $\mathcal{M}, \mathcal{M}^{\prime}$ with planar faces are parallel if they are combinatorially equivalent, and corresponding edges are parallel.

Multilayer constructions. Structures like those schematically depicted by Figure 2 include not only one mesh, but several meshes, corresponding to the different layers of the construction. It is natural to demand that meshes which correspond to different layers are parallel.

Geometrically optimal nodes. In the actual realization of a polyhedral surface $\mathcal{M}$ as a steel/glass roof, planar glass panels are held by prismatic beams following the edges of $\mathcal{M}$ (see Fig. 4). A beam is symmetric with respect to its central plane which passes through the edge corresponding to the beam. A node corresponds to a vertex $\mathbf{m}_{i}$ and connects incoming beams in a way which supports the force flow imposed by the overall statics of the structure. Node construction and manufacturing are greatly simplified if there is a node axis $A_{i}$, which is contained in the central planes of incoming beams (see Figure 4, left). Figure 4, right, shows the case of a welded node which does not have a node axis. Obviously the handling and manufacturing of such 'nodes with torsion' is more complicated than the case with a node axis. Geometrically, lines $A_{i}$ passing through the vertices $\mathbf{m}_{i}$ of a given mesh are a collection of node axes, if and only if

$$
\mathbf{m}_{i} \mathbf{m}_{j} \text { is an edge } \Longrightarrow \text { node axes } A_{i}, A_{j} \text { are co-planar. }
$$

To avoid pathological cases, we forbid that node axes lie in edges.

The following simple but fundamental proposition establishes a property of a collection of node axes associated with a mesh $\mathcal{M}$. It relates node axes to an auxiliary mesh $\mathcal{M}^{\prime}$ which is parallel to the given mesh, and is illustrated by Fig. 4, left.

Proposition 1 If the meshes $\mathcal{M}, \mathcal{M}^{\prime}$ with vertices $\mathbf{m}_{i}, \mathbf{m}_{i}^{\prime}(\forall i$ : $\mathbf{m}_{i} \neq \mathbf{m}_{i}^{\prime}$ ) are parallel, then the lines $A_{i}=\mathbf{m}_{i} \vee \mathbf{m}_{i}^{\prime}$ serve as node axes for the mesh $\mathcal{M}$ (provided no line $A_{i}$ contains an edge).

Conversely assume that a simply connected mesh $\mathcal{M}$ is equipped with node axes $A_{i}$ passing through its vertices $\mathbf{m}_{i}$. Then there exists a mesh $\mathcal{M}^{\prime}$ parallel to $\mathcal{M}$, such that $A_{i}$ is spanned by corresponding vertices $\mathbf{m}_{i}, \mathbf{m}_{i}^{\prime}$.

Proof: This is shown in [Pottmann et al. 2007]. Part (i) is elementary. For part (ii) we start with a vertex $\mathbf{m}_{i_{0}}^{\prime} \in A_{i_{0}}$ and construct further vertices of $\mathcal{M}^{\prime}$ by the requirement that corresponding edges of $\mathcal{M}$ and $\mathcal{M}^{\prime}$ are parallel, and that $\mathbf{m}_{i}^{\prime} \in A_{i}$ for all $i$.

Geometric support structure. There is a point of view which unifies the different cases where parallel meshes occur - regardless of whether a parallel mesh is physically realized, or is only used in the definition of node axes. This point of view is that we emphasize the construction elements which connect different layers - physically present or not - and which are transverse to the mesh $\mathcal{M}$ under consideration. In Figure 2, right, these construction elements are the glass panels which connect the two parallel meshes $\mathcal{M}, \mathcal{M}^{\prime}$. In Figure 4, left, these construction elements are the beams. We shrink those elements until they have zero width (this is schematically indicated in Fig. 4, left). They then become

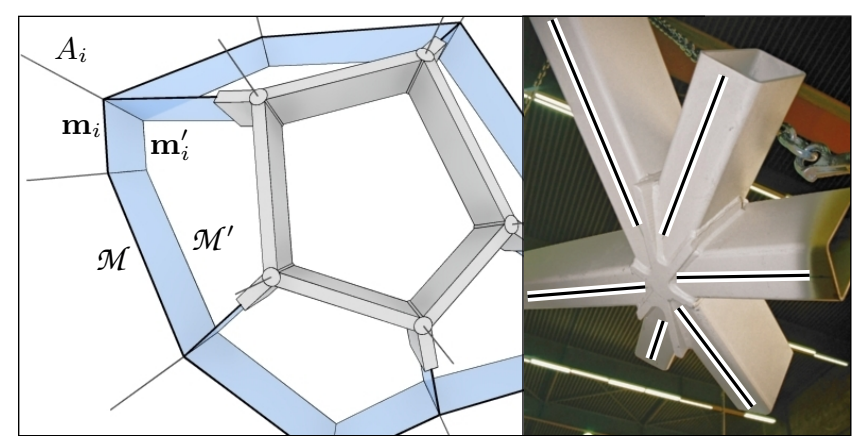

Figure 4: Left: This figure shows nodes, supporting beams, and the underlying geometric support structure of a steel/glass construction, based on a mesh $\mathcal{M}$ (vertices $\mathbf{m}_{i}$ ) and its parallel mesh $\mathcal{M}^{\prime}$ (vertices $\left.\mathbf{m}_{i}^{\prime}\right)$. All beams are symmetric with respect to their central plane (blue); at an optimized (torsion-free) node $\mathbf{m}_{i}$ the central planes of supporting beams pass through the node axis $A_{i}=\mathbf{m}_{i} \vee \mathbf{m}_{i}^{\prime}$. Right: A node without axis, with geometric torsion.

planar quadrilaterals transverse to the mesh $\mathcal{M}$, passing through the edges of $\mathcal{M}$. Those transverse quads which are adjacent to a node $\mathbf{m}_{i}$, have a common edge which lies in the node axis $A_{i}$. Such a collection of quads is called a geometric support structure of the mesh $\mathcal{M}$ (see Figures 1, 2, 4, 6, 13, and 14).

Obviously, a collection of node axes for $\mathcal{M}$ almost uniquely defines the quads of a geometric support structure for $\mathcal{M}$ - the only degree of freedom left is the quad boundaries opposite to the edges of $\mathcal{M}$. By Proposition 1, any geometric support structure joins the edges of $\mathcal{M}$ with the respective corresponding edges of a mesh $\mathcal{M}^{\prime}$ parallel to $\mathcal{M}$. Every node axis $A_{i}$ joins corresponding vertices $\mathbf{m}_{i}, \mathbf{m}_{i}^{\prime}$.

Specific problems considered in this paper. We are going to discuss meshes with certain geometric properties relevant to architectural design, especially support structures. An important property of this kind is that a mesh possesses a support structure whose beams are of constant height - this is the class of edge offset meshes defined later. Generally speaking, the more properties we require a mesh to have, the fewer degrees of freedom are available. We therefore encounter the following problems:

- The approximation problem. Is it possible to approximate a given shape by a mesh contained in a specific class of meshes? For example, this is pssible for the conical quad meshes, as shown by Liu et al. [2006], but not for the quad meshes with the edge offset property. However we shall see that we can approximate arbitrary surfaces if we relax the requirement of constant height a little bit.

- The design problem. How can we explore or even completely describe the set of meshes with a specific geometric property? This question is especially important in cases where the approximation problem is not solvable. For example, we will introduce some geometric transformations which change shape but preserve the edge offset property.

\subsection{Basics of mesh parallelism}

Mesh parallelism and the spaces $\mathcal{C}(\mathcal{M})$ and $\mathcal{P}(\mathcal{M})$. A mesh $\mathcal{M}$ is represented by the list $\left(\mathbf{m}_{1}, \ldots \mathbf{m}_{N}\right) \in \mathbb{R}^{3 N}$ of vertices and the mesh combinatorics, i.e., the information which vertices belong to common edges and faces. We use $\mathcal{C}(\mathcal{M})$ to denote the linear $3 N$-dimensional space of meshes combinatorially equivalent to $\mathcal{M}$. If $\mathcal{M}^{\prime}, \mathcal{M}^{\prime \prime}$ have the same combinatorics, a linear combination $\lambda^{\prime} \mathcal{M}^{\prime}+\lambda^{\prime \prime} \mathcal{M}^{\prime \prime}$ is defined vertex-wise; this operation corresponds to the linear combination of vectors in $\mathbb{R}^{3 N}$. 


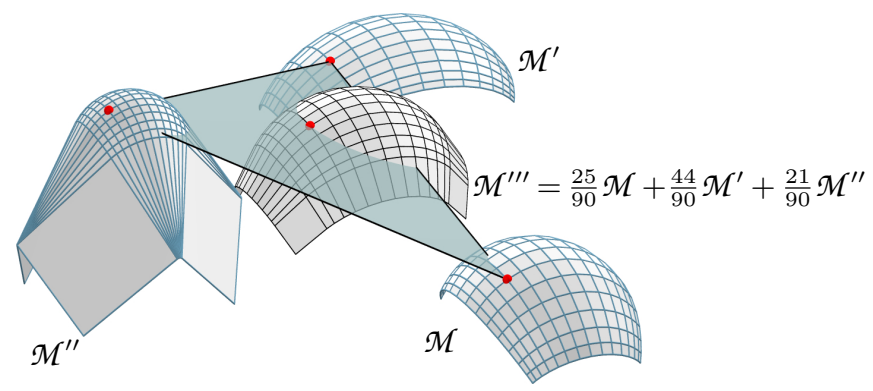

Figure 5: The set $\mathcal{P}(\mathcal{M})$ of meshes parallel to a given mesh $\mathcal{M}$ is a linear space and can be explored by a linear blend of some of its elements.

Meshes $\mathcal{M}, \mathcal{M}^{\prime}$ are parallel, if $\mathcal{M}^{\prime} \in \mathcal{C}(\mathcal{M})$ and corresponding edges are parallel (see Figures 3 and 5 ). We use that definition only if the faces of $\mathcal{M}$ are planar. Clearly then corresponding faces of $\mathcal{M}$ and $\mathcal{M}^{\prime}$ lie in parallel planes (parallelity of planes alone is sufficient to guarentee parallelity of edges, if no pair of adjacent faces are co-planar). We denote the set of meshes parallel to $\mathcal{M}$ by $\mathcal{P}(\mathcal{M})$. To avoid pathological cases we require that the mesh $\mathcal{M}$ which defines the space $\mathcal{P}(\mathcal{M})$ has only nonzero edges. Trivial ways of producing meshes parallel to $\mathcal{M}$ are to translate and scale $\mathcal{M}$. Since triangles with parallel edges are scaled copies of each other, two parallel triangle meshes are scaled copies of each other [Pottmann et al. 2007]. This is the reason why we do not consider triangle meshes in the rest of the paper.

Suppose $\mathcal{M}^{\prime}, \mathcal{M}^{\prime \prime} \in \mathcal{P}(\mathcal{M})$. Then, for each edge $\mathbf{m}_{i} \mathbf{m}_{j}$, the vectors $\mathbf{m}_{i}^{\prime}-\mathbf{m}_{j}^{\prime}, \mathbf{m}_{i}^{\prime \prime}-\mathbf{m}_{j}^{\prime \prime}$ are multiples of $\mathbf{m}_{i}-\mathbf{m}_{j}$. It follows that any expression $\left(\lambda^{\prime} \mathbf{m}_{i}^{\prime}+\lambda^{\prime \prime} \mathbf{m}_{i}^{\prime \prime}\right)-\left(\lambda^{\prime} \mathbf{m}_{j}^{\prime}+\lambda^{\prime \prime} \mathbf{m}_{j}^{\prime \prime}\right)$ is a multiple of $\mathbf{m}_{i}-\mathbf{m}_{j}$. This shows that the linear combination $\lambda^{\prime} \mathcal{M}^{\prime}+\lambda^{\prime \prime} \mathcal{M}^{\prime \prime}$ is also parallel to $\mathcal{M}$, so $\mathcal{P}(\mathcal{M})$ is a linear subspace of $\mathcal{C}(\mathcal{M})$. The zero vector of both $\mathcal{P}(\mathcal{M})$ and $\mathcal{C}(\mathcal{M})$ is the mesh $\mathbf{o}=0 \cdot \mathcal{M}$, all of whose vertices coincide with the origin of the coordinate system. Linear blending between three meshes in the space $\mathcal{P}(\mathcal{M})$ is illustrated by Figure 5 .

The space $\mathcal{P}(\mathcal{M})$ does not only contain 'nice' meshes. We may see various undesirable effects such as unevenly distributed faces, sharp edges of regression, or overlapping regions. Nevertheless, it is both theoretically and practically useful to have the entire space $\mathcal{P}(\mathcal{M})$ at our disposal. Even visually unpleasant meshes in $\mathcal{P}(\mathcal{M})$ will turn out to be helpful in the computation of optimal beam layouts (see Fig. 14d).

Computing in the space of parallel meshes. As $\mathcal{P}(\mathcal{M})$ is a linear space, it is important to determine a basis. We observe that a mesh $\mathcal{M}^{\prime} \in \mathcal{C}(\mathcal{M})$ is contained in $\mathcal{P}(\mathcal{M})$ if and only if

$$
\mathbf{m}_{i} \mathbf{m}_{j} \text { is an edge } \Longrightarrow \mathbf{m}_{i}^{\prime}-\mathbf{m}_{j}^{\prime}=\lambda_{i j}\left(\mathbf{m}_{i}-\mathbf{m}_{j}\right) .
$$

We can therefore determine $\mathcal{P}(\mathcal{M})$ as the solution space of the system of equations

$$
\left(\mathbf{m}_{i}^{\prime}-\mathbf{m}_{j}^{\prime}\right) \times\left(\mathbf{m}_{i}-\mathbf{m}_{j}\right)=0 \quad \text { for all edges } \mathbf{m}_{i} \mathbf{m}_{j} .
$$

A rough count of degrees of freedom (one d.o.f. per edge, two closure conditions per face, 3 d.o.f. for the translations in space) shows that e.g. for open meshes we can expect $\operatorname{dim} \mathcal{P}(\mathcal{M})=$ \# edges $-2 \times$ \# faces +3 . The actual solution of (2) is done via SVD. Thresholding of small singular values is supported by an a priori estimate for $\operatorname{dim} \mathcal{P}(\mathcal{M})$.

Once a basis is available, the minimization of linear and quadratic functionals (e.g. a fairness functional) under constraints (like fixed points) and linear side conditions (like edge length inequalities) presents no problems.

\section{Offset meshes}

Meshes are offsets of each other, if they are parallel, and in addition their distance from each other is constant throughout the mesh. This notion is similar to the concept of smooth offset surfaces [Maekawa 1999], but in contrast to the smooth case, for meshes there are various different definitions of distance. Consequently, there are also various different notions of offset mesh. Two of them, face offsets and edge offsets are relevant to problems of architectural design of freeform surfaces considered in this paper.

For multilayer constructions (cf. Figure 2) it is natural to require that the distance of corresponding faces is constant. Parallel meshes with this property (face offsets) are the topic of [Liu et al. 2006].

Another type of offset occurs when we employ parallel meshes for the layout of beams in a steel/glass construction based on a given mesh $\mathcal{M}$. The beams are a physical realization of a geometric support structure which connects two parallel meshes $\mathcal{M}$ and $\mathcal{M}^{\prime}$. If the distance of corresponding edges in $\mathcal{M}$ and $\mathcal{M}^{\prime}$ is constant throughout the mesh, then beams of constant height are perfectly aligned on both the upper (outer) and the lower (inner) side of the construction, provided that the mesh is convex (see Figure 6).

If, on the other hand, the distance of corresponding edges in $\mathcal{M}$ and $\mathcal{M}^{\prime}$ is not the same throughout the mesh, then one could still use beams with the same cross-section throughout the mesh, but these beams will not be aligned on both sides (see Figure 7). Still, if $\mathcal{M}$ and $\mathcal{M}^{\prime}$ are at almost constant edge-edge distance, a physical realization may use beams of the same cross-section throughout the mesh, without too much misalignment being visible. As exact distance requirements are sometimes hard or impossible to fulfill, we consider offsets at an exactly constant distance (in this section) as well as offsets at approximately constant distance (in Section 4).

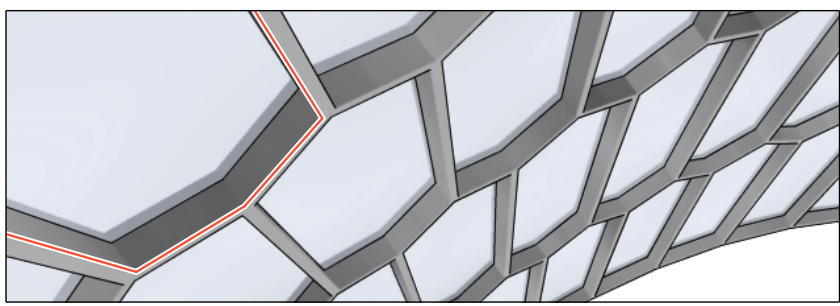

Figure 6: This construction (a detail of Fig. 1) is based on an edge offset mesh and has beams of constant height. In the positively curved areas, edges (red) of beams with rectangular cross-section have an exact intersection at the nodes, which follows from Prop. 3.

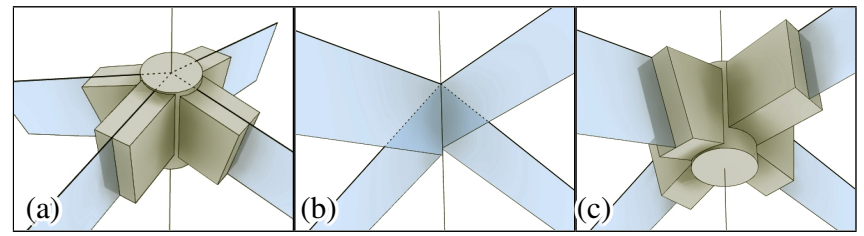

Figure 7: This geometric support structure is defined by two parallel meshes which are not of constant edge-edge distance. We nevertheless employ beams of constant height to physically realize that support structure. The resulting misalignment is not visible from the outside (a), hardly visible from the beams' mid-sections lying in the respective central planes (b), but is clearly visible from the inside (c). Still, nodes have no torsion and symmetry planes of beams intersect in the node axis.

ACM Transactions on Graphics, Vol. 26, No. 3, Article 65, Publication date: July 2007. 


\subsection{Types of exact offset meshes}

Recall that a mesh $\mathcal{M}^{\prime} \in \mathcal{P}(\mathcal{M})$ at constant distance from $\mathcal{M}$ is an offset of $\mathcal{M}$. Different ways to define the precise meaning of "dist $\left(\mathcal{M}, \mathcal{M}^{\prime}\right)=d$ " lead to different kinds of offsets:

- vertex offsets: The distance of corresponding vertices $\mathbf{m}_{i}, \mathbf{m}_{i}^{\prime}$ equals a constant $d$, which does not depend on the vertex.

- edge offsets: The distance of corresponding parallel edges (actually, lines which carry those edges) does not depend on the edge and equals $d$.

- face offsets: The distance of faces (actually, planes which carry faces) is independent of the face and equals $d$.

Discrete Gauss images. If $\mathbf{p}$ is a point of a smooth surface and $\mathbf{n}$ is the unit normal vector there, then $\mathbf{p}^{\prime}=\mathbf{p}+d \mathbf{n}$ would be a point of an offset surface at distance $d$. If $\mathbf{p}, \mathbf{p}^{\prime}$ are given, we can recover the unit normal vector by $\mathbf{n}=\left(\mathbf{p}^{\prime}-\mathbf{p}\right) / d$. If $\mathcal{M}^{\prime}$ is an offset mesh of $\mathcal{M}$ we can mimick this construction and define a discrete Gauss image mesh $\mathcal{S}:=\left(\mathcal{M}^{\prime}-\mathcal{M}\right) / d$, whose vertices $\mathbf{s}_{i}=\left(\mathbf{m}_{i}^{\prime}-\mathbf{m}_{i}\right) / d$ can be regarded as discrete normal vectors.

Proposition 2 Consider a mesh $\mathcal{M}$, its offset mesh $\mathcal{M}^{\prime}$ at distance $d$, and define the Gauss image mesh $\mathcal{S}=\left(\mathcal{M}^{\prime}-\mathcal{M}\right) / d$. Then the following is true:

1. $\mathcal{M}^{\prime}$ is a vertex offset of $\mathcal{M} \Longleftrightarrow$ the vertices of $\mathcal{S}$ are contained in the unit sphere $S^{2}$. If $S$ is a quad mesh and no edges degenerate, then $\mathcal{M}$ has a vertex offset if and only if $\mathcal{M}$ is a circular mesh, i.e., each face has a circumcircle.

2. $\mathcal{M}^{\prime}$ is an edge offset of $\mathcal{M} \Longleftrightarrow$ the edges of the Gauss image mesh $S$ are tangent to $S^{2}$.

3. $\mathcal{M}^{\prime}$ is a face offset of $\mathcal{M} \Longleftrightarrow$ the faces of the Gauss image mesh $\mathcal{S}$ are tangent to $S^{2}$. A mesh has a face offset if and only if it is conical, i.e., the faces around a vertex are tangent to a cone of revolution.

So in all three cases we have the equivalence $\operatorname{dist}\left(\mathcal{M}, \mathcal{M}^{\prime}\right)=d$ $\Longleftrightarrow \operatorname{dist}(S, \mathbf{o})=1$, which means that the vertices, or the edges, or the faces of $\mathcal{S}$ are at distance 1 from the origin.

Proof: The equivalence $\operatorname{dist}\left(\mathcal{M}^{\prime}, \mathcal{M}\right)=d \Longleftrightarrow \operatorname{dist}(\mathcal{S}, \mathbf{o})=1$ is elementary. The statements about circular and conical meshes are reviewed in [Pottmann and Wallner 2007].

This relation between a pair of offset meshes $\mathcal{M}, \mathcal{M}^{\prime}$ and the Gauss image mesh $\mathcal{S}$ is illustrated by Figure 8. Proposition 2 has an important consequence: If the mesh $\mathcal{M}$ has an offset mesh at constant vertex/edge/face distance, then every mesh parallel to $\mathcal{M}$ has this property. This is because the Gauss image mesh $S \in \mathcal{P}(\mathcal{M})$ can
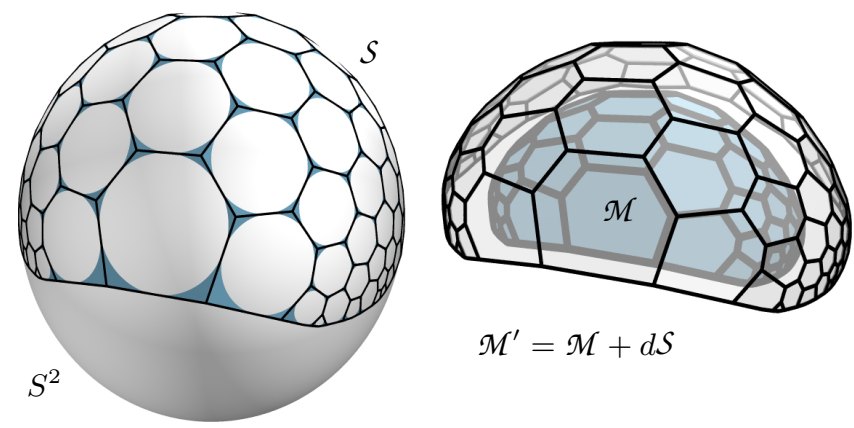

$$
\mathcal{M}^{\prime}=\mathcal{M}+d \mathcal{S}
$$

Figure 8: A mesh $\mathcal{M}$ with an edge offset mesh $\mathcal{M}^{\prime}$ at distance $d$ has a parallel mesh $S=\left(\mathcal{M}^{\prime}-\mathscr{M}\right) / d$ whose edges are tangent to the unit sphere $S^{2}$. The faces of $S$ intersect $S^{2}$ in a circle packing, cf. Section 3.2. be used to construct an offset not only for $\mathcal{M}$, but for any further mesh in $\mathcal{P}(\mathcal{M})$. Another observation will be important later: We can first construct a mesh whose vertices/edges/faces are at distance 1 from the origin. Then any mesh $\mathcal{M} \in \mathcal{P}(S)$ has offset meshes $\mathcal{M}^{\prime}=\mathcal{M}+d \mathcal{S}$.

\subsection{Meshes with edge offsets}

We are interested in meshes which have edge offsets (EO meshes) because they can be built with beams of constant height meeting at the nodes in a geometrically optimal way (see Figure 6). Proposition 2 mentioned that a mesh $\mathcal{M}$ has an edge offset mesh, if there is a mesh $\mathcal{S}$ parallel to $\mathcal{M}$ whose edges are tangent to the unit sphere. The following paragraphs deal with the interesting mathematical theory of EO meshes, with a focus on the geometry of $\mathcal{S}$.

Proposition 3 If a mesh $\mathcal{M}$ has an edge offset $\mathcal{M}^{\prime}$, then for each vertex $\mathbf{m}_{i}$ of $\mathcal{M}$, the edges emanating from $\mathbf{m}_{i}$ are contained in a cone of revolution $\Gamma_{i}$. The node axis $A_{i}$ spanned by corresponding vertices $\mathbf{m}_{i} \in \mathcal{M}, \mathbf{m}_{i}^{\prime} \in \mathcal{M}^{\prime}$ is the axis of the cone $\Gamma_{i}$.

Proof: The statement about cones is true for the mesh $\mathcal{M}$ if and only if it is true for at least one mesh in $\mathcal{P}(\mathcal{M})$ which does not have zero edges (because corresponding edges are parallel). It is thus sufficient to show it for the Gauss image mesh $S=\left(\mathcal{M}^{\prime}-\mathcal{M}\right) / d$, where $d=\operatorname{dist}\left(\mathcal{M}, \mathcal{M}^{\prime}\right)$. According to Proposition 2, the edges of $\mathcal{S}$ are tangent to the unit sphere $S^{2}$ (see Figure 8 and especially Figure 9). Obviously, all lines emanating from a vertex $\mathbf{s}_{i}$ which touch $S^{2}$ lie in a cone of revolution $\widetilde{\Gamma}_{i}$, so the statement is true for $\mathcal{S}$. Consequently it is true for $\mathcal{M}$. The axis of $\widetilde{\Gamma}_{i}$ passes through the origin, so it is parallel to the vector $\mathbf{s}_{i}$. It follows that the axis $A_{i}$ of the cone $\Gamma_{i}$ associated with the vertex $\mathbf{m}_{i}$ contains the point $\mathbf{m}_{i}^{\prime}=\mathbf{m}_{i}+d \mathbf{s}_{i}$.
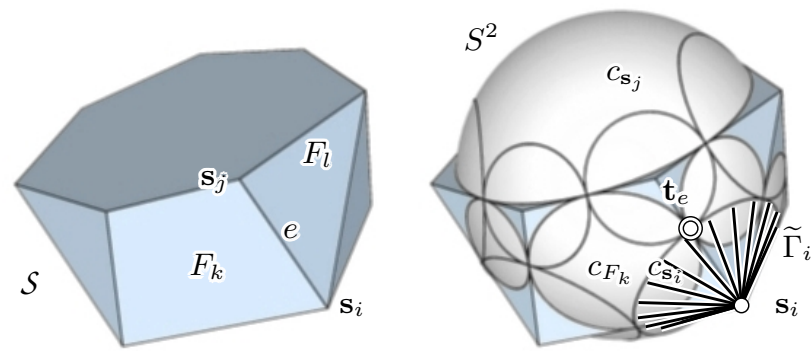

Figure 9: A Koebe polyhedron and related circles and cones.

EO meshes and Koebe polyhedra. A mesh $S$ with planar faces whose edges $e$ touch $S^{2}$ in points $\mathbf{t}_{e}$ (a so-called Koebe polyhedron) has very interesting geometry [Bobenko and Springborn 2004; Ziegler 1995]. Each face $F$ intersects $S^{2}$ in a circle $c_{F}$ which touches the boundary edges of $F$ from the inside (see Figures 8 and 9). For any vertex $\mathbf{s}_{i}$, the vertex cone $\widetilde{\Gamma}_{i}$ touches the unit sphere in a circle $c_{\mathbf{s}_{i}}$. Obviously the edge $e$ has a point of tangency $\mathbf{t}_{e}$ with $S^{2}$, and two circles of either type pass through $\mathbf{t}_{e}$. Circles of the same type touch each other, and circles of different types intersect at 90 degrees. The computation of such circle patterns via minimization of a convex function is known [Bobenko and Springborn 2004] and even possible on-line [Sechelmann 2006]. Closed Koebe polyhedra are uniquely determined by their combinatorics up to a Möbius transformation (i.e., a projective mapping which transforms $S^{2}$ into itself). For open polyhedra there is an additional degree of freedom for each boundary vertex. 

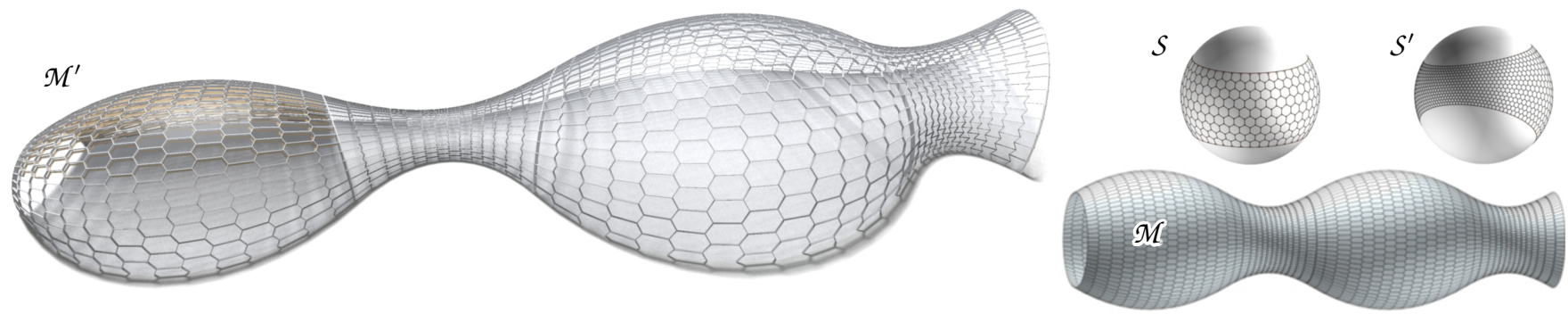

Figure 10: Creation of the mesh $\mathcal{M}^{\prime}$ which Figure 1 is based on. This example demonstrates that meshes with properties interesting from the mathematical viewpoint can yield aesthetically pleasing results; and that a designer has access to additional degrees of freedom by applying some nonstandard geometric transformations. Here we start with the Koebe polyhedron $\mathcal{S}$ and construct the mesh $\mathcal{M}$ which is of constant mean curvature with respect to $\mathcal{S}$ (the Gauss mapping $\sigma: \mathcal{M} \rightarrow S$ has an overfolding, with the inflection circle on $\mathcal{M}$ corresponding to the apparent boundary of $\mathcal{S}$ ). Applying a Laguerre transformation results in the EO mesh $\mathcal{M}^{\prime}$, which has the Gauss image mesh $\mathcal{S}^{\prime}$. This L-transform was found interactively.

\subsection{Designing with EO meshes}

The edge offset property is rather restrictive. Quad-dominant meshes which have vertex or face offsets (i.e., the circular and conical meshes), are capable of approximating arbitrary shapes. This is no longer the case with EO meshes.

Computing EO meshes from Koebe polyhedra. We may use the following general procedure when designing a mesh $\mathcal{M}$ with the edge offset property: First we determine the combinatorics of the mesh and compute a Koebe polyhedron $S$ with that combinatorics, using the procedure of [Bobenko and Springborn 2004]. The mesh $\mathcal{M}$ we are looking for is then found within the space $\mathcal{P}(\mathcal{S})$, e.g. by optimization. An example of this is shown by Figure 11, where $\mathcal{S}$ is a Koebe polyhedron with pentagonal faces and $\mathcal{M}$ is found by minimizing the fairness functional $f_{\text {Laplacian }}$ defined by

$$
f_{\text {Laplacian }}(\mathcal{M})=\sum_{\text {vertices } \mathbf{m}_{i}}\left(\mathbf{m}_{i}-\frac{1}{\operatorname{deg}\left(\mathbf{m}_{i}\right)} \sum_{\mathbf{m}_{j} \in \operatorname{star}\left(\mathbf{m}_{i}\right)} \mathbf{m}_{j}\right)^{2}
$$

under appropriate sign constraints for the factors $\lambda_{i j}$ of Equ. (1) (see the figure caption for more details). Once an EO mesh is found, we may apply geometric transformations to it - this is the topic of the next paragraph. It is obvious that these methods are not useful for geometric modeling in the usual sense, but only for form finding purposes.

Laguerre transformations of EO meshes. From the various equivalent descriptions of Laguerre geometry [Cecil 1992], the following, which employs the spheres of $\mathbb{R}^{3}$ as basic elements, is perhaps shortest: A sphere $S$ with center $\left(m_{1}, m_{2}, m_{3}\right)$ and signed radius $r$ is identified with the point $\mathbf{x}_{S}=\left(m_{1}, m_{2}, m_{3}, r\right) \in \mathbb{R}^{4}$. We think of normal vectors of spheres pointing to the outside if and only if $r>0$. Points are spheres of zero radius. An $L$-transformation then has the form $\mathbf{x}_{S} \mapsto A \cdot \mathbf{x}_{S}+\mathbf{a}$, where $\mathbf{a} \in \mathbb{R}^{4}$ and $A$ is a $4 \times 4$ matrix with $A^{T} J A=J$ and $J=\operatorname{diag}(1,1,1,-1)$. Every Euclidean transform permutes the set of spheres and can be written as an L-transform. Another simple example of an L-transform ( $A=I_{4}$ and $\mathbf{a}=(0,0,0, d)$ ) is the offsetting operation which increases the radius by the value $d$. It is well known that the set of spheres tangent to an oriented cone of revolution is mapped by any L-transform $\alpha$ to a set of the same type [Cecil 1992]. Thus, an oriented cone of revolution $\Gamma$ becomes an entity of Laguerre geometry: Take two spheres $S_{1}, S_{2}$ tangent to $\Gamma$ and define $\alpha(\Gamma)$ to be tangent to the spheres $\alpha\left(S_{1}\right), \alpha\left(S_{2}\right)$. After these preparations we can state:

Proposition 4 An L-transformation maps an edge offset mesh $\mathcal{M}$ to another edge offset mesh $\mathcal{M}^{\prime}$, if both are seen as the respective collection of vertex cones $\Gamma_{i}, \Gamma_{i}^{\prime}$ according to Proposition 3.
The proof is not difficult, but exceeds the scope of this paper. We use Proposition 4 for the modification of edge offset meshes. An example is furnished by the mesh Figure 1 is based on; the transformation we use is illustrated by Figure 10.

Possible shapes of EO meshes (quad and hex mesh cases). For a mesh $\mathcal{M}$ with the edge offset property, the Gauss image mesh $S$ is a Koebe polyhedron. Bobenko et al. [2006] show that in case of quad meshes, $S$ is a so-called s-isothermic mesh and thus the mesh $\mathcal{M}$ is a discrete variant of a curvature line parametrization whose Gauss image is an isothermic curve network. Such "L-isothermic surfaces" are mentioned by Blaschke [1929], but not much seems to be known about their shapes. Likewise, the description of the possible shapes obtainable by quadrilateral EO meshes is an unsolved problem at the present time.

Hexagonal meshes, which here is a synonym for meshes with planar faces and vertices of valence three, have better approximation properties (cf. e.g. [Cutler and Whiting 2007]). In order to create a hexagonal EO Mesh $\mathcal{M}$ which approximates a given surface $\Phi$, we could start with any Koebe polyhedron $S$ which is a hexagonal mesh (cf. Figures 10 and 18) and determine the vertices $\mathbf{m}_{i}$ of $\mathcal{M}$ as follows: Parallel translate the three planes which are adjacent to the vertex $\mathbf{s}_{i}$ in the mesh $\mathcal{S}$ so that they touch $\Phi$, and intersect them. By

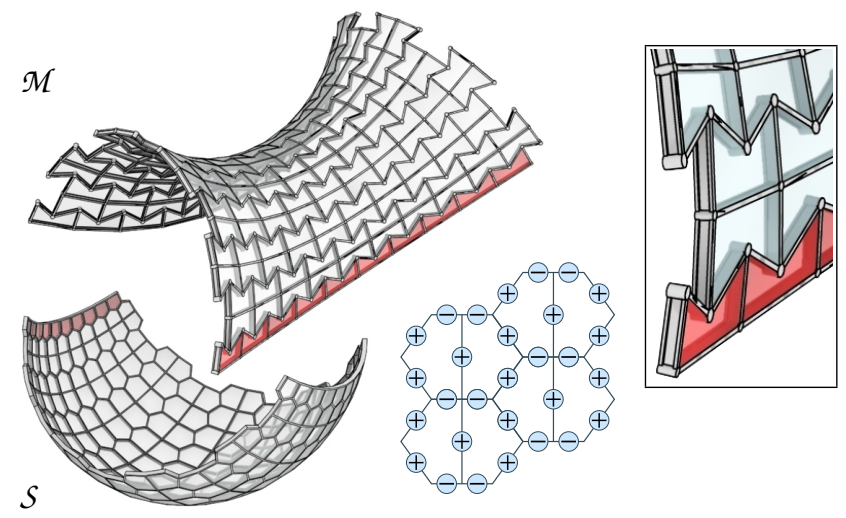

Figure 11: Edge offset mesh of negative curvature. The mesh $\mathcal{M}$ with pentagonal faces has a parallel mesh $\mathcal{S}$ whose edges are tangent to the unit sphere, so $\mathcal{M}$ has the edge offset property. Corresponding edges of $\mathcal{M}$ and $\mathcal{S}$ are parallel, but the correspondence is orientation-reversing for some edges. The general pattern which edges keep their orientation and which do not is indicated by the schematic diagram. The mesh $\mathcal{S}$ is a Koebe polyhedron; $\mathcal{M}$ was found by minimizing the Laplacian energy of Equation (3) in the space $\mathcal{P}(S)$, under appropriate sign constraints on the coefficients $\lambda_{i j}$ of Equation (1).

ACM Transactions on Graphics, Vol. 26, No. 3, Article 65, Publication date: July 2007. 
construction, $\mathcal{M}$ has the edge offset property and the planes which carry its faces touch $\Phi$. Unfortunately it is apparently difficult to guarantee that $\mathcal{M}$ is a nice polyhedral surface without self-intersections, so much work remains to be done before such a procedure can be used as an effective design tool. The present paper does not enter the topic of approximating general surfaces with hex meshes.

\section{Optimizing support structures}

In view of Proposition 2 we cannot expect a general mesh with planar faces which is neither circular nor conical to have vertex offsets or face offsets. The class of meshes with edge offsets is even more restricted, as discussed in Sections 3.2 and 3.3. Therefore the problem of constructing offsets at approximately constant distance is important. This section first discusses such approximate offset meshes from a theoretical viewpoint, and then shows how to compute them by minimizing a quadratic function in the space $\mathcal{P}(\mathcal{M})$. We also treat the problem of finding a mesh with planar faces which approximates a given shape in the first place. This completes the processing pipeline from shape to mesh, and further to support structure. The last part of this section deals with more complex optimization problems.

\subsection{Approximate offsets}

The mesh $\mathcal{M}^{\prime}$ which is parallel to the mesh $\mathcal{M}$ is said to be an approximate offset of $\mathcal{M}$, if $\mathcal{M}^{\prime}=\mathcal{M}+d \mathcal{S}$, where $\mathcal{S} \in \mathcal{P}(\mathcal{M})$ is a mesh which approximates the unit sphere. We say that the distance of $\mathcal{M}^{\prime}$ from $\mathcal{M}$ is approximately constant, and that $S$ is an approximate Gauss image of $\mathcal{M}$. As there are different kinds of (exact) Gauss image anyway, we will drop the attribute 'approximate' and simply speak of a Gauss image. The vertex $\mathbf{s}_{i}$ of $\mathcal{S}$ corresponding to a vertex $\mathbf{m}_{i}$ of $\mathcal{M}$ is considerd as an approximate normal vector for the vertex $\mathbf{m}_{i}$. Then the approximate offset at distance $d$ has vertices $\mathbf{m}_{i}+d \mathbf{s}_{i}$, which is directly analogous to the previous notation (see Figure 12). We use the symbol $\sigma: \mathcal{M} \rightarrow \mathcal{S}$ for the natural correspondence between the meshes $\mathcal{M}$ and $\mathcal{S}$ ( $\sigma$ is the Gauss mapping). The computation of an approximate Gauss image for a given mesh with planar faces is discussed in Sections 4.2 and 4.3 below.

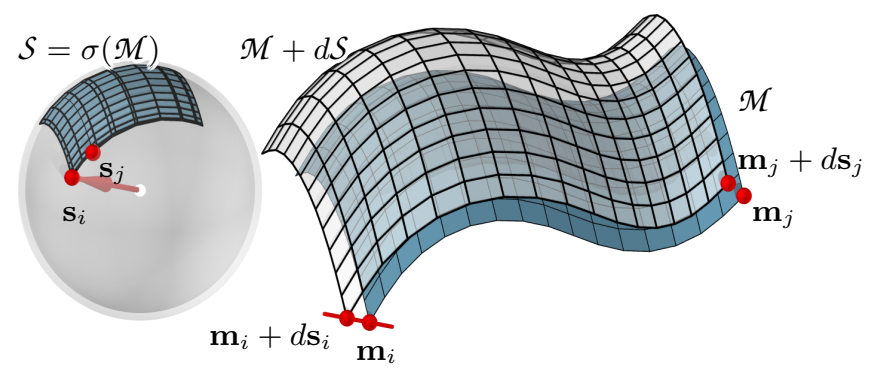

Figure 12: This figure illustrates how we assign a geometric support structure to a given mesh $\mathcal{M}$ and thus make it buildable with optimized nodes and controlled beam heights. Optimization in the space $\mathcal{P}(\mathcal{M})$ yields a parallel mesh $\mathcal{S}$ approximating the unit sphere $S^{2}$, thus defining offsets $\mathcal{M}^{\prime}=\mathcal{M}+d \mathcal{S}$ at approximately constant distance $d$. Here $\mathcal{S}$ has an overfolding due to a change in the sign of curvature in $\mathcal{M}$, and is contained in the layer between radii 0.98 and 1.04 .

\subsection{Offset meshes by optimization in $\mathcal{P}(\mathcal{M})$}

We approach the problem of computing offsets at approximately constant distance as follows: For a given mesh $\mathcal{M}$ we must find a mesh $\mathcal{S} \in \mathcal{P}(\mathcal{M})$ which approximates the unit sphere ( $\mathcal{S}$ is a Gauss image of $\mathcal{M}$ ). We can convert this problem into minimization of a quadratic functional: For each face $F$, we have its normal vector $\mathbf{n}_{F}$, and a vertex $\mathbf{m}_{i(F)} \in F$. For each vertex $\mathbf{m}_{i}$, we estimate a unit normal vector $\widetilde{\mathbf{n}}_{i}$. The vertex of $S$ corresponding to the vertex $\mathbf{m}_{i}$ is denoted by $\mathbf{s}_{i}$. We set up the functionals

$f_{\text {faces }}=\sum_{\text {faces } F}\left(\mathbf{n}_{F} \cdot\left(\mathbf{s}_{i(F)}-\mathbf{n}_{F}\right)\right)^{2}, f_{\text {vert }}=\sum_{\text {vertices } \mathbf{m}_{i}}\left(\mathbf{s}_{i}-\widetilde{\mathbf{n}}_{i}\right)^{2}$,

and minimize a linear combination of $f_{\text {faces }}, f_{\text {vert }}$, and the fairness functional $f_{\text {Laplacian }}$ of Equation (3). The aims $f_{\text {vert }} \rightarrow$ min and $f_{\text {faces }} \rightarrow$ min express the requirement that indeed the mesh $S$ is an (approximate) Gauss image of $\mathcal{M}$. Results are shown by Figures 12 and 13. Also the processing pipeline described in Section 4.3 uses this construction (cf. Figure 14). We may assign a low weight to the fairness term for $\mathcal{S}$, as a lack of fairness for $\mathcal{S}$ is hardly noticeable in the support structure (Fig. 14d). It may be more important that beam heights are approximately constant.

\subsection{A processing pipeline from shape to beam layout}

Section 4.2 describes how we can find, for a given mesh $\mathcal{M}$ with planar faces, an approximate offset, which can be used e.g. for the definition of beams of approximately constant heights. This section considers also the problem which in the overall processing pipeline comes earlier: How to find a mesh which has planar faces and approximates a given shape.

Liu et al. [2006] compute a quad-dominant mesh with planar faces for a given surface by optimizing a mesh which follows a network of conjugate curves (of which Figures 14, a-c show some examples). Obviously the angle of intersection of such curves is important for the quality of the mesh, but for negatively curved surfaces it is not guaranteed that a network of conjugate curves has only transverse intersections. The special case of principal curvature lines has an intersection angle of $90^{\circ}$ throughout the network. However, we would like to have more degrees of freedom at our disposal when choosing the curve network.

Figure 14 illustrates our approach to this problem. We apply an affine transformation $\alpha$ to the given surface, compute principal curvature lines for the transformed surface, and transform them back with $\alpha^{-1}$. Conjugacy of curves is invariant with respect to affine transformations, and the intersection angle of the resulting curves is close to $90^{\circ}$ if $\alpha$ is close to the identity transformation. So we are able to create a number of suitable curve networks simply by choosing different affine mappings (see Figures 14, a-c).

We then lay out a quad-dominant mesh along a curve network which fits the design intent best, e.g. has few singularities, or has the singularities in the right places (we choose Figure 14c). This mesh is optimized such that its faces become planar, using the procedure employed by Liu et al. [2006]. Having computed the mesh $\mathcal{M}$, we next need to find a support structure with beams of approximately constant height. This is done by finding a mesh $\mathcal{S} \in \mathcal{P}(\mathcal{M})$ which approximates $S^{2}$, using the minimization procedure described in Section 4.2. A result is shown by Figure 14d. The geometric support structure which is bounded by the meshes $\mathcal{M}$ and $\mathcal{M}^{\prime}=\mathcal{M}+d S$ is illustrated by Figure $14 \mathrm{e}$.

\subsection{Other ways of optimization}

Combined optimization of mesh and Gauss image. Section 4.2 discussed the problem of computing a geometric support structure - or an approximate Gauss image $\mathcal{S}$ - for a given mesh $\mathcal{M}$, and in that section we described how to solve that problem by quadratic optimization in the space $\mathcal{P}(\mathcal{M})$. Here we go one step further and optimize both $\mathcal{M}$ and $S$ at the same time. 
(a)

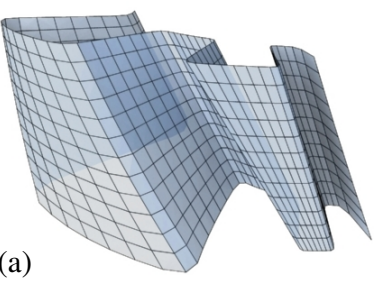

(b)

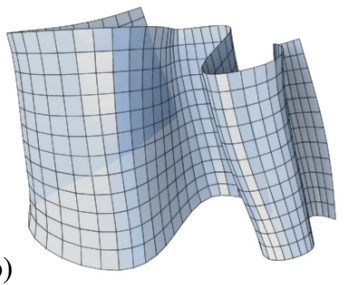

(c)

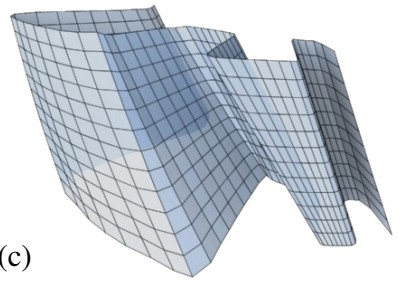

(d)

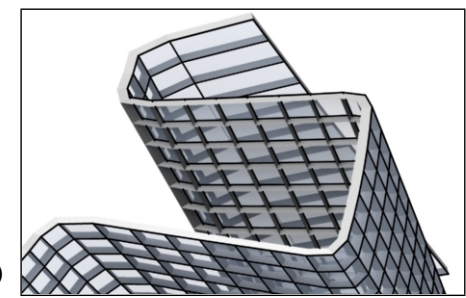

Figure 13: This figure illustrates that finding a support structure according to the method of Section 4.2 can be done with little change in the original mesh. (a) a mesh $\mathcal{M}$ created by subdivision. (b) In order to create a geometric support structure with nice offset properties, we first follow [Liu et al. 2006] and make the mesh conical by perturbing vertices. As the intersection angles of mesh polylines in $\mathcal{M}$ are far from 90 degrees, the optimized mesh deviates much from $\mathcal{M}$. (c) If $\mathcal{M}$ is optimized to become planar, not necessarily conical, the deviations from the original mesh are small. $(d)$ We apply our procedure for creating a support structure with approximately constant beam heights.

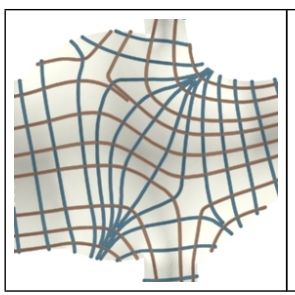

(a)

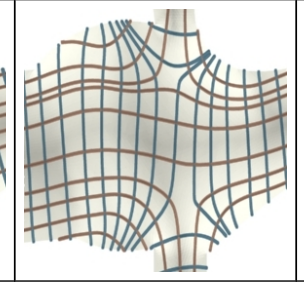

(b)

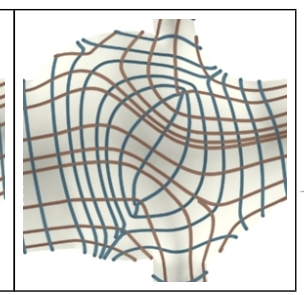

(c)

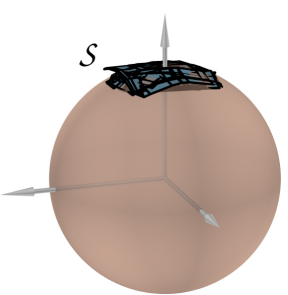

(d)

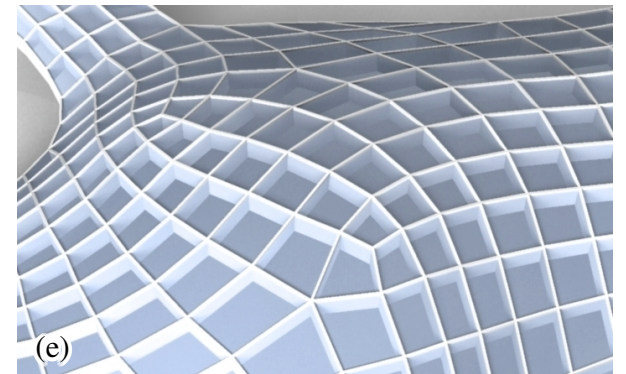

Figure 14: Meshing and construction of a support structure with optimized nodes for a given architectural freeform design. (a)-(c). A study of different conjugate curve networks is performed. We lay out a quad mesh $\mathcal{M}$ along the network in (c) and use the method of [Liu et al. 2006] to optimize $\mathcal{M}$ such that its faces become planar. $\mathcal{M}$ is shown in subfigure (e). (d) We recompute a Gauss image $\mathcal{S}$ of $\mathcal{M}$ which approximates the unit sphere $S^{2}$. (e) $\mathcal{S}$ leads to a support structure with optimized nodes and approximately equal beam heights for $\mathcal{M}$.

The purpose of this computation is to design a mesh which has offset or curvature properties useful for architectural design. We know that any such mesh has to approximately follow a network of principal curves. We start with a mesh with this property and set up an optimization problem as follows. We consider the functionals $f_{\text {close }, 1}:=\sum_{i} \operatorname{dist}\left(\mathbf{m}_{i}, \Phi\right)^{2}$ and $f_{\text {close }, 2}:=\sum_{i}\left\|\mathbf{s}_{i}\right\|^{2}$, which express proximity of the vertices of $\mathcal{M}, \mathcal{S}$ to their respective reference surfaces $\Phi, S^{2}$; further, the functional

$$
f_{\text {par }}:=\sum_{\text {edges } \mathbf{m}_{i} \mathbf{m}_{j}}\left\|\frac{\mathbf{m}_{i}-\mathbf{m}_{j}}{\left\|\mathbf{m}_{i}-\mathbf{m}_{j}\right\|} \times\left(\mathbf{s}_{i}-\mathbf{s}_{j}\right)\right\|^{2},
$$

which expresses parallelity of meshes $\mathcal{M}$ and $\mathcal{S}$; the fairness functionals $f_{\text {Laplacian }}(\mathcal{M}), f_{\text {Laplacian }}(\mathcal{S})$ according to Equation (3); and the functional $f_{\text {det }}$ of [Liu et al. 2006] which expresses planarity of the mesh $\mathcal{M}$. We then use a penalty method to minimize a linear combination of the functionals above. Other functionals may be included. An example which includes a functional aiming at constant mean curvature is presented in Section 5 (Figure 19).

Methods of optimization and numerics. The solution of the nonlinear optimization problems which arise when minimizing the functionals above, under the side condition of planarity of faces and parallelity of meshes, usually is difficult. We have employed a penalty method analogous to the mesh optimization procedure described by [Liu et al. 2006]; actually the problems to be solved in the present context are similar to that paper. In order to minimize a functional $F$ under $k$ different constraints $G_{1}=0, \ldots, G_{k}=0$, we consider the auxiliary minimization problem $\lambda F+\sum \mu_{j} G_{j}^{2} \rightarrow$ min: For stable convergence, $\lambda$ has a higher value at the beginning, and tends to zero as optimization progresses. The unconstrained minimization problem $\lambda F+\sum \mu_{j} G_{j}^{2} \rightarrow$ min can effectively be solved by the Gauss-Newton method with LM regularization [Kelley 1999]. In our case, $F$ is a linear combination of $f_{\text {close }, 1}, f_{\text {close }, 2}$, $f_{\text {Laplacian }}(\mathcal{M}), f_{\text {Laplacian }}(S)$, while the constraints are given by $f_{\text {det }}$ and $f_{\text {par }}$. This method usually requires user interaction when balancing the weights of the individual functionals.

Translating the Gauss image. We want to mention a simple fact which nevertheless has an interesting application: If $S$ is a Gauss image for the mesh $\mathcal{M}$, then formally any translate $S^{\prime}=S+\mathbf{x}$ is a valid Gauss image, simply because it was never specified how well $\mathcal{S}^{\prime}$ must approximate the unit sphere, and meshes $\mathcal{S}^{\prime}$ and $\mathcal{M}$ are parallel. It is a different question if we can still call the vertices of $\mathcal{S}^{\prime}$ normal vectors of the polyhedral surface $\mathcal{M}$ without violating geometric intuition, but if $\mathrm{x}$ is small, we surely can (see Fig. 15).

Figure 1 shows an architectural design based on a mesh $\mathcal{M}$ and two different geometric support structures: One is defined by a Gauss image mesh $S$ whose edges are tangent to $S^{2}$. It is used to create the supporting beams (then of constant height). The other one is based on the Gauss image $\mathcal{S}^{\prime}=S+\mathrm{x}$, and is physically realized as shading elements. The vector $\mathbf{x}$ has been found by subjecting it to optimization: We select parallel light (for particular sun positions) and compute $\mathbf{x}$ such that the total area of shadow cast by the shading elements is maximal, under the constraint $\|\mathbf{x}\| \leq 0.99$.
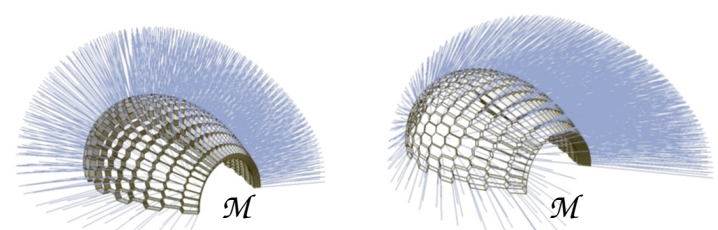

Figure 15: These two systems of 'approximate normal vectors' of a mesh $\mathcal{M}$ are defined by a Gauss image $\mathcal{S}$, whose edges are tangent to $S^{2}$ (left image), and the Gauss image $\mathcal{S}+\mathbf{x}$ (right image). They correspond to the two different support structures employed in Fig. 1.

ACM Transactions on Graphics, Vol. 26, No. 3, Article 65, Publication date: July 2007. 


\section{Curvatures in meshes with planar faces}

It has been observed many times that properties of smooth or discrete surfaces which are interesting from the mathematical viewpoint often lead to very aesthetic figures [Sullivan 2005]. This is even more important in our current work which focuses on architecture and design. Therefore the aim of this section on curvatures is not only to create geometric functionals useful for smoothing and other optimization tasks, but to lay the foundations for interactive design and form finding tools. We want to demonstrate that the earlier theory developed in the paper, namely offset properties and support structures based on parallelism, is compatible with the second one, namely the definition of curvatures based on mesh parallelism. Not only they are compatible, but surfaces of constant or vanishing mean curvature, as well as other special surfaces, serve as basis of architectural designs with functional properties.

Preparation: Mixed areas and Steiner's formula. Assume that $P=\left(\mathbf{p}_{0}, \ldots, \mathbf{p}_{k-1}\right)$ and $Q=\left(\mathbf{q}_{0}, \ldots, \mathbf{q}_{k-1}\right)$ are planar polygons whose corresponding edges are parallel ('parallel polygons'). Then also the polygon $P+d Q=\left(\mathbf{p}_{0}+d \mathbf{q}_{0}, \ldots\right)$ is parallel to $P$ and $Q$. We are interested in the oriented area of $P+d Q$, with the orientation defined by a normal vector $\mathbf{n}$ of the plane which contains $P$. Its computation is based on the formula $\frac{1}{2} \operatorname{det}(\mathbf{b}-\mathbf{a}, \mathbf{c}-\mathbf{a}, \mathbf{n})$ for the area of a triangle with vertices $\mathbf{a}, \mathbf{b}, \mathbf{c}$, lying in a plane with unit normal vector $\mathbf{n}$. It follows that $\operatorname{area}(P+d Q)=\frac{1}{2} \sum_{i=0}^{k-1} \operatorname{det}\left(\mathbf{p}_{i}+d \mathbf{q}_{i}, \mathbf{p}_{i+1}+d \mathbf{q}_{i+1}, \mathbf{n}\right)$, with indices modulo $k$. This implies that

$$
\begin{aligned}
& \operatorname{area}(P+d Q)=\operatorname{area}(P)+2 d \operatorname{area}(P, Q)+d^{2} \operatorname{area}(Q), \text { with } \\
& \operatorname{area}(P, Q)=\frac{1}{4} \sum_{i=0}^{k-1}\left[\operatorname{det}\left(\mathbf{p}_{i}, \mathbf{q}_{i+1}, \mathbf{n}\right)+\operatorname{det}\left(\mathbf{q}_{i}, \mathbf{p}_{i+1}, \mathbf{n}\right)\right] .
\end{aligned}
$$

The computation so far is well known $-\operatorname{area}(P, Q)$ denotes the mixed area of polygons $P$ and $Q$ in the terminology of convex geometry [Schneider 1993].

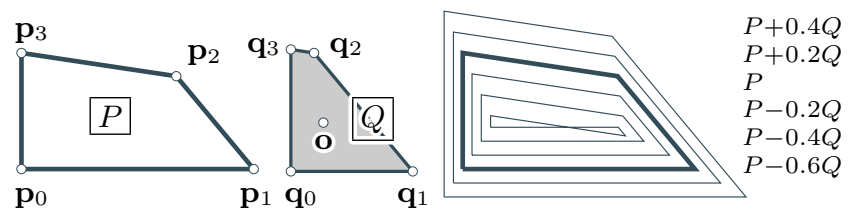

Figure 16: Parallel polygons with vertices $\mathbf{p}_{i}, \mathbf{q}_{i}$, and $\mathbf{p}_{i}+d \mathbf{q}_{i}$.

Curvatures of faces. Curvatures in polyhedral surfaces can be defined in different ways. One may be guided by the idea that a discrete surface approximates a smooth one, and define a curvature by way of numerical differentiation. Another method is to observe relations between curvatures and geometric properties in smooth surfaces, and to postulate an analogous relation for the discrete case, like in the definition of the mean curvature vector by Polthier [2002a] as the gradient of the area functional for triangle meshes. In our setting we consider the variation of surface area when passing from a surface $\Phi$ to an offset surface $\Phi^{d}$ : Each point $\mathbf{x} \in \Phi$ is moved to $\mathbf{x}+d \mathbf{n}(\mathbf{x})$, where " $\mathbf{n}$ " is the field of unit normal vectors. Then the surface area changes according to

$$
\operatorname{area}\left(\Phi^{d}\right)=\int_{\Phi}\left(1-2 d H(\mathbf{x})+d^{2} K(\mathbf{x})\right) d \mathbf{x},
$$

with $H$ as mean and $K$ as Gaussian curvature (Steiner's formula). In the discrete case the change in area exhibits a quite similar behaviour:

Proposition 5 The surface area of the (approximate) offset $\mathcal{M}^{d}=$ $\mathcal{M}+d S$ of the mesh $\mathcal{M}$ w.r.t. to the Gauss image mesh $\sigma(\mathcal{M})=\mathcal{S}$
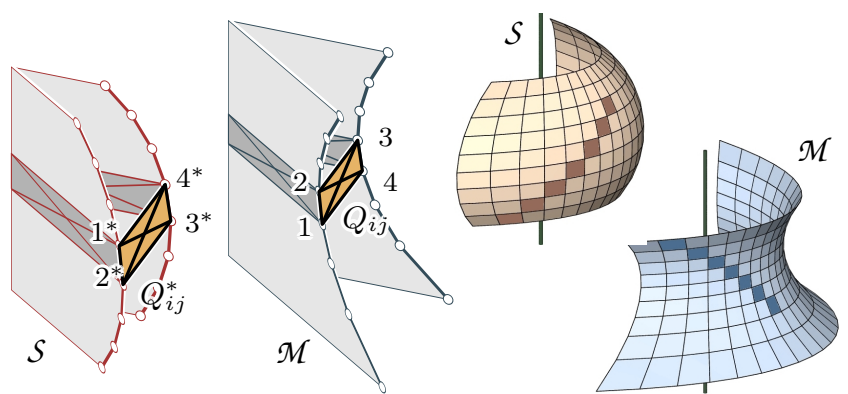

Figure 17: Construction of simple minimal quad meshes via parallelity of diagonals. The meshes $\mathcal{M}, \mathcal{S}$ carry 'horizontal' polylines in horizontal planes and 'meridian polylines' in planes through a fixed axis. $\mathcal{S}, \mathcal{M}$ are parallel - note corresponding faces $Q_{i j}$ and $Q_{i j}^{*}-$ but the correspondence is orientation-reversing. The mean curvature of the face $Q_{i j}$ in the mesh $\mathcal{M}$ with respect to the Gauss image $\mathcal{S}$ vanishes if and only if diagonals in $Q_{i j}$ are parallel to diagonals in $Q_{i j}^{*}\left(13 \| 2^{*} 4^{*}\right.$ and $\left.24 \| 1^{*} 3^{*}\right)$.

obeys the law

$$
\begin{gathered}
\operatorname{area}\left(\mathcal{M}^{d}\right)=\sum_{F: \text { face of } \mathcal{M}}\left(1-2 d H_{F}+d^{2} K_{F}\right) \text { area }(F), \text { with } \\
H_{F}=-\frac{\operatorname{area}(F, \sigma(F))}{\operatorname{area}(F)}, \quad K_{F}=\frac{\operatorname{area}(\sigma(F))}{\operatorname{area}(F)} .
\end{gathered}
$$

Here each face $F$ of $\mathcal{M}$ is oriented such that area $(F)>0$.

Proof: It is sufficient to show (6) and (7) for a single face $F$. This follows directly from (4) by comparing coefficients.

When we compare Equations (5) and (6), we see that it is natural to define mean curvature and Gaussian curvature of the face $F$ by the quantities $H_{F}$ and $K_{F}$ given by (7). This definition of curvatures does not refer to the mesh $\mathcal{M}$ alone, but implicitly assumes that the Gauss image mesh $\mathcal{S}$ is given. The values of $H_{F}$ and $K_{F}$ behave exactly like they should also in other respects. One is that the Gaussian curvature is the quotient of areas between Gauss image and original surface, as in the smooth case. Another one is that for most faces $F$, meaningful principal curvatures $\kappa_{1, F}, \kappa_{2, F}$ can be defined, such that

$$
H_{F}=\left(\kappa_{1, F}+\kappa_{2, F}\right) / 2, \quad K_{F}=\kappa_{1, F} \kappa_{2, F} .
$$

The curvatures $\kappa_{1, F}, \kappa_{2, F}$ are the roots of the polynomial $f(x)=$ $x^{2}-2 H_{F} x+K_{F}$; they exist if and only if

$$
H_{F}^{2}-K_{F} \geq 0 \text {. }
$$

We do not give details, but it is not difficult to show that this inequality is true whenever the face $F$, or its Gauss image $\sigma(F)$ is strictly convex. This follows from Minkowski's first inequality "area $(P, Q)^{2}-\operatorname{area}(Q) \operatorname{area}(P) \geq 0$ ", which applies when both $P, Q$ are convex [Schneider 1993].

Meshes of constant mean curvature. Discrete surfaces of constant mean curvature $H_{F}$ (cmc meshes) or discrete minimal surfaces, which have $H_{F}=0$, are interesting not only from the purely mathematical viewpoint, but also from the viewpoint of aesthetics. The condition that a mesh $\mathcal{M}$ has constant curvatures with respect to a Gauss image mesh $\mathcal{S}$ is not as rigid as one might expect, and it is possible to construct a great variety of such meshes (both quadrilateral and hexagonal), even meshes with the edge offset property.

First we discuss minimal meshes. The condition of minimality means that for all faces $F$, we have $H_{F}=0$, and consequently the parallel polygons $F$ and $\sigma(F)$ have vanishing mixed area. It is 


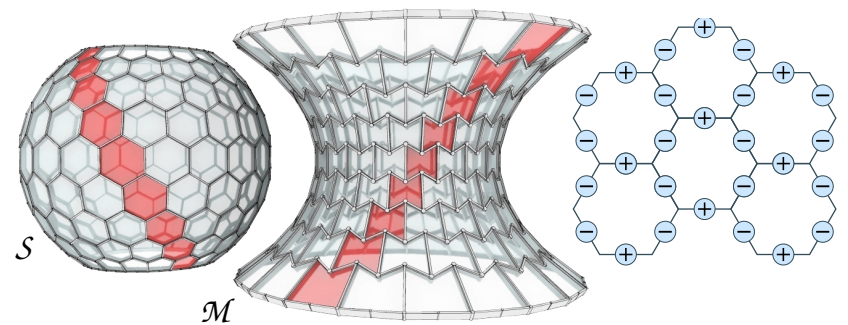

Figure 18: A discrete minimal EO mesh. The mesh $\mathcal{M}$ is constructed from the Koebe polyhedron $\mathcal{S}$ by the conditions that $\mathcal{M}$ and $\mathcal{S}$ are parallel meshes, and that the mixed areas of all corresponding faces vanish. Both $\mathcal{M}$ and $\mathcal{S}$ have rotational symmetry. The correspondence between $\mathcal{M}$ and $\mathcal{S}$ is orientation-reversing for some edges; the sign pattern is schematically illustrated at right.

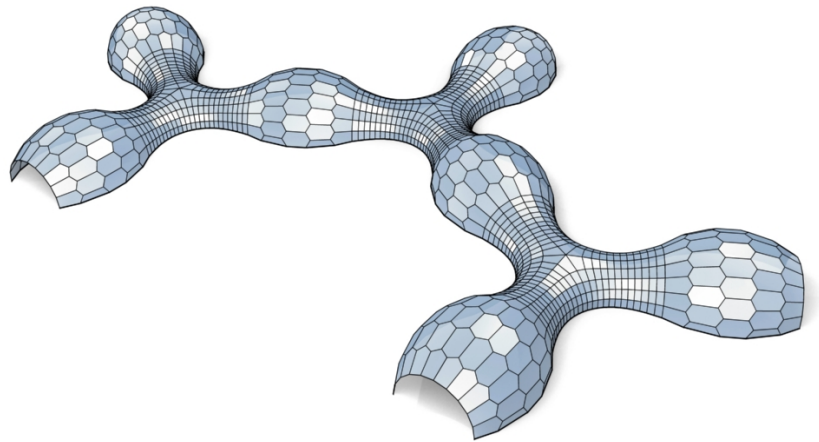

Figure 19: This design with convex faces is composed from pieces of discrete cmc surfaces obtained in different ways. The junction piece (quad mesh) was originally computed as a trinoid (cf. [Grosse-Brauckmann and Polthier 1997; Schmitt 2004]) by the applet at [Schmitt 2003]. After combined optimization of mesh and Gauss image in order to achieve planarity of faces and $H_{F}=$ const., we arrive at a planar quad mesh which has $H_{F} \in[0.966,1.048]$. The bulging pieces are hexagonal EO meshes whose mean curvature with respect to a previously chosen Koebe polyhedron as Gauss image is exactly constant.

easy to show that in the case of parallel quadrilaterals $Q=1234$ and $Q^{*}=1^{*} 2^{*} 3^{*} 4^{*}$, we have

$$
\operatorname{area}\left(Q, Q^{*}\right)=0 \Longleftrightarrow 13 \text { parallel } 2^{*} 4^{*}, 24 \text { parallel } 1^{*} 3^{*}
$$

(see Figure 17). It is worth noting that this parallelity of diagonals in corresponding quads also occurs in the Christoffel duality constructions of [Bobenko and Pinkall 1996] and [Bobenko et al. 2006], which has gone unnoticed so far. For this reason we would like to call parallel meshes Christoffel transforms of each other, if corresponding faces have vanishing mixed area.

We should note that not every mesh $S$ has the property that there exists a mesh $\mathcal{M}$ which is minimal with respect to $\mathcal{S}$ as Gauss image. In the following we therefore restrict ourselves to special cases.

Example: minimal and cmc quad meshes of simple geometry. For quad meshes $\mathcal{M}$ and $\mathcal{S}$ of 'generalized rotational symmetry' as described by Fig. 17, we can construct a minimal mesh $\mathcal{M}$ for given $S$ by starting with one vertex, say the one denoted by " 1 ", and computing the faces of $\mathcal{M}$ step by step. They are uniquely determined by the requirements of parallelity of edges and parallelity of diagonals. The construction of a cmc mesh $\mathcal{M}$ from $\mathcal{S}$ is quite analogous to the minimal surface case; instead of the condition $H_{F}=0$ we have now $H_{F}=$ const (we omit the details) Surfaces of revolution where the vertices of the Gauss image mesh $\mathcal{S}$ lie in the unit sphere (and therefore $\mathcal{M}$ is a circular mesh), have been considered by [Hoffmann 1998].
Example: Hexagonal meshes of rotational symmetry which have vanishing or constant mean curvature. The previous example concerning quad meshes extends to hex meshes as well, if we restrict ourselves to meshes with rotational symmetry (see Figures 10, 18, and 19). We do not provide details here, because they are not difficult and would take up too much space. We only mention that by splitting symmetric hexagons into quads we can treat this case in a way very similar to the previous example.

\section{Discussion}

Limitations. With highly nonlinear optimization problems, there is in general no guarantee that optimization achieves success and is not stuck in a local minimum. Therefore it is very important to know beforehand which meshes can be optimized towards the goal under consideration. E.g. if a quad-dominant mesh is to become planar by moving vertices as little as possible, then this mesh must originally have been aligned with a conjugate network of curves [Liu et al. 2006]. We did not experience problems when the original mesh was chosen appropriately. However, this issue is very important for applications in practice. We also emphasized on easier optimization tasks like optimization in $\mathcal{P}(\mathcal{M})$ for computing a support structure, which exhibit quite tame behaviour.

The complexity of the modeling task shown by Figure 14 is rather high. It is hard to satisfy all design requirements if the underlying reference surface is not very smooth. This problem becomes even more severe if boundary conditions have to be met. As a consequence it would be difficult to find a geometrically optimal support structure for data sets like the Stanford bunny, for instance. Fortunately, architectural designs tend to be smoother.

Implementation and run times. The most computationally expensive tasks in the present paper are nonlinear optimization procedures, for which we employed a Gauss-Newton method, and computing a basis of $\mathcal{P}(\mathcal{M})$ which is done by SVD. SVD runs well even if it needs a long run time, because we estimate the dimension of $\mathcal{P}(\mathcal{M})$ beforehand. The run times of code on a $2 \mathrm{GHz} \mathrm{PC}$ with 1 GB RAM are as follows: Computation of principal curves in Fig. 14a-c (not a topic of the present paper) and meshing costs 25 seconds each. The resulting mesh $\mathcal{M}$ has 649 vertices and 568 faces. Planarization costs 3 seconds, and a basis of $\mathcal{P}(\mathcal{M})$ is computed with SVD in 4.4 minutes; the Gauss image of Fig. 14d takes 0.68 seconds to compute. As to Fig. 19, simultaneous optimization of the mesh and its Gauss image towards $H=$ const. needs 3 minutes.

Conclusion. We have presented mesh parallelism as a basic method for the solution of problems which occur in the design and construction of architectural freeform structures. It allows us to find an optimized beam layout with torsion free nodes, even after the design phase when we are just given a mesh with planar faces. Moreover, it is a key tool for modeling meshes with special offset properties. We introduced the new class of edge offset meshes which yield the cleanest possible nodes, if built with beams of constant height. Our results apply to EO quad meshes as well as to the more flexible pentagonal and hexagonal meshes. As a contribution to aesthetic design and a component for geometric optimization algorithms, we introduced a novel discrete curvature theory which is based on parallel meshes. In our examples we have pointed to invariance under certain transformations, which turned out to be of great practical value (blending of meshes, Laguerre transformations).

Future research. There is plenty of possibilities for future research, and we want to mention just a few directions. The discrete curvature theory presented here possesses many more extensions of the classical theory than could be described here. They will be 
published in a forthcoming paper. A particularly interesting topic is the new type of Christoffel duality which applies to exactly those planar quad meshes whose diagonal meshes can be brought into static equilibrium. This will result in new insights on discrete minimal surfaces and cmc surfaces, and thus deliver new interesting shapes useful in particular for architectural design. Future research should also address aesthetic meshing of given shapes, for both the quad mesh and hex mesh cases. In general, the interactive design of meshes with functional properties relevant to architecture is a topic of high interest.

Acknowledgments. This work has been performed in cooperation with Waagner-Biro Stahlbau, who have wide expertise in the manufacturing of freeform steel/glass architecture. We are grateful to be able to use real data for visualization of our algorithms in Fig. 14. The authors would like to thank H. Schmiedhofer for his implementation of geometric support structures in architectural CAD software and his help in the preparation of figures. This work was supported by grants No. 19214-N18 and S9206-N12 of the Austrian Science Fund (FWF). The work of Yang Liu and Wenping Wang was partially supported by the National Key Basic Research Project of China under 2004CB318000. The work of Alexander Bobenko was partially supported by the DFG Research Group "Polyhedral Surfaces" and the DFG Research Center MATHEON "Mathematics for key technologies" in Berlin. Last, but not least, our thanks go to the anonymous referees for their extensive comments.

\section{References}

Akleman, E., Srinivasan, V., And Mandal, E. 2005. Remeshing schemes for semi-regular tilings. In Shape Modeling and Applications, Proceedings. IEEE, 44-50.

Alliez, P., Cohen-Steiner, D., Devillers, O., Levy, B., AND DESBRUN, M. 2003. Anisotropic polygonal remeshing. ACM Trans. Graphics 22, 3, 485-493.

BlASCHKE, W. 1929. Vorlesungen über Differentialgeometrie, vol. 3. Springer.

Bobenko, A., And Pinkall, U. 1996. Discrete isothermic surfaces. J. Reine Angew. Math. 475, 187-208.

Bobenko, A., AND Springborn, B. 2004. Variational principles for circle patterns and Koebe's theorem. Trans. Amer. Math. Soc. 356, 659-689.

Bobenko, A., And Suris, Yu., 2005. Discrete differential geometry. Consistency as integrability. Monograph pre-published at http://www.arxiv.org/math/0504358.

Bobenko, A., And SURIS, Yu. 2007. On organizing principles of discrete differential geometry, geometry of spheres. Russian Math. Surveys 62, 1, 1-43.

Bobenko, A., Hoffmann, T., And Springborn, B. 2006. Minimal surfaces from circle patterns: Geometry from combinatorics. Ann. of Math. 164, 231-264.

Brell-Cokcan, S., And Pottmann, H., 2006. Tragstruktur für Freiformflächen in Bauwerken. Patent No. A1049/2006.

CECIL, T. 1992. Lie Sphere Geometry. Springer.

Cohen-Steiner, D., Alliez, P., And Desbrun, M. 2004. Variational shape approximation. ACM Trans. Graphics 23, 3, 905-914.

Cutler, B., And Whiting, E. 2007. Constrained planar remeshing for architecture. In Proc. Graphics Interface.
Glymph, J., Shelden, D., Ceccato, C., Mussel, J., And SCHOBER, H. 2002. A parametric strategy for freeform glass structures using quadrilateral planar facets. In Acadia 2002, ACM, 303-321.

Grosse-Brauckmann, K., And Polthier, K. 1997. Constant mean curvature surfaces derived from Delaunay's and Wente's examples. In Visualization and mathematics. Springer, 119-134.

Hoffmann, T. 1998. Discrete rotational CMC surfaces and the elliptic billiard. In Mathematical Visualization, H. C. Hege and K. Polthier, Eds. Springer, 117-124.

KELLEY, C. T. 1999. Iterative Methods for Optimization. SIAM.

LiU, Y., Pottmann, H., Wallner, J., Yang, Y.-L., and WANG, W. 2006. Geometric modeling with conical meshes and developable surfaces. ACM Trans. Graphics 25, 3, 681-689.

MAEKAWA, T. 1999. An overview of offset curves and surfaces. Computer-Aided Design 31, 251-267.

NISHIKAWA, Y., ET AL. 1998. Measurements of interfacial curvatures of bicontinuous structure from three-dimensional digital images. 1. A parallel surface method. Langmuir 14, 1241-1249.

Oswald, P., AND SCHröDER, P. 2003. Composite primal/dual $\sqrt{3}$-subdivision schemes. Comp. Aid. Geom. Des. 20, 135-164.

Polthier, K. 2002. Polyhedral surfaces of constant mean curvature. Habilitationsschrift TU Berlin.

Polthier, K. 2002. Unstable periodic discrete minimal surfaces. In Nonlinear Partial Differential Equations. Springer, 127-143.

PotTmann, H., AND Wallner, J. 2007. The focal geometry of circular and conical meshes. Adv. Comput. Math. to appear.

Pottmann, H., BRell-CoKCAn, S., ANd Wallner, J. 2007. Discrete surfaces for architectural design. In Curves and Surfaces: Avignon 2006, P. Chenin et al., Eds. Nashboro Press.

SAUER, R. 1970. Differenzengeometrie. Springer.

SCHIEF, W. K. 2006. On a maximum principle for minimal surfaces and their integrable discrete counterparts. J. Geom. Physics $56,1484-1495$.

SchmitT, N., 2003. Noid. Java Applet, http://www-sfb288.math. tu-berlin.de/ nick/Noid/NoidApplet.html.

SCHMitT, N., 2004. Constant mean curvature trinoids. preprint, http://www.arxiv.org/math/0403036.

SCHNEIDER, R. 1993. Convex bodies: the Brunn-Minkowski theory. Cambridge University Press.

Schober, H. 2003. Freeform glass structures. In Glass Processing Days 2003. Glass Processing Days, Tampere (Fin.), 46-50.

Schramm, O. 1997. Circle patterns with the combinatorics of the square grid. Duke Math. J. 86, 347-389.

Sechelmann, S., 2006. Koebe polyhedron editor. Java Applet, http://www.math.tu-berlin.de/geometrie/ps/software.shtml.

SG. The smart geometry group. http://www.smartgeometry.com.

Sullivan, J. 2005. The aesthetic value of optimal geometry. In The Visual Mind II, M. Emmer, Ed. MIT Press, 547-563.

Tong, Y., Alliez, P., Cohen-Steiner, D., And Desbrun, M. 2006. Designing quadrangulations with discrete harmonic forms. In Symp. Geometry Processing. 201-210.

ZIEGLER, G. 1995. Lectures on Polytopes. Springer. 
